Revue européenne des sciences sociales

European Journal of Social Sciences

54-1 | 2016

Les usages sociaux des sciences du vivant

\title{
Johan HEILBRON, French Sociology
}

\section{Christian Papilloud}

\section{OpenEdition}

\section{Journals}

Édition électronique

URL : http://journals.openedition.org/ress/3383

DOI : $10.4000 /$ ress.3383

ISBN : $1663-4446$

ISSN : 1663-4446

Éditeur

Librairie Droz

Édition imprimée

Date de publication : 15 mai 2016

Pagination : 263-266

ISSN : 0048-8046

Référence électronique

Christian Papilloud, « Johan HEILBRON, French Sociology», Revue européenne des sciences sociales [En ligne], 54-1 | 2016, mis en ligne le 15 mai 2016, consulté le 22 septembre 2020. URL : http:// journals.openedition.org/ress/3383; DOI : https://doi.org/10.4000/ress.3383

Ce document a été généré automatiquement le 22 septembre 2020

(C) Librairie Droz 


\title{
Johan HEILBRON, French Sociology
}

\author{
Christian Papilloud
}

\section{RÉFÉRENCE}

Johan HEILBRON, 2015, French Sociology, Ithaca \& London, Cornell University Press, $272 \mathrm{p}$.

1 Ce livre est l'aboutissement d'un travail de longue haleine mené depuis les années 1980 sur la sociologie française, des fondateurs jusqu'aux sociologues contemporains que Johan Heilbron a côtoyés. Il comprend une introduction, sept chapitres, une conclusion et un épilogue. Heilbron utilise la théorie des champs de Pierre Bourdieu qui donne à son exposé un caractère pédagogique affirmé (p. 5).

2 1) "L'établissement d'une science sociale organisée ». Au XIX siècle, la sociologie française naît au croisement des sciences humaines. Ces trois contextes influencent autant ses méthodes que son style d'écriture (p. 33). L'organisation des sciences sociales en France est marquée par divers conflits - notamment la critique du positivisme et le recours au néo-kantisme. Les premiers sociologues ne se contentent toutefois pas de réagir à ces débats, ils proposent aussi des alternatives originales qui contribuent à enrichir le champ des sciences sociales (p. 37).

2) «Une science improbable ». Heilbron revient sur la contribution d'Auguste Comte, l'inventeur du mot sociologie dans les années 1830 (p.38). Comte autonomise la discipline des théories qui la précèdent dans un contexte où la France domine le monde intellectuel. S'il se fit autant d'ennemis que d'amis, il n'en contribue pas moins à la "seconde révolution scientifique» (p.42). Cette dernière se caractérise par l'institutionnalisation de nombreuses disciplines qui entrent à l'Université, processus dont le Cours de philosophie positive représente la théorisation. Heilbron relève également l'importance de Frédéric Le Play, des premières études sur la situation sociale des familles en Europe et d'une littérature sociale marquée par le marxisme et les débats sur le socialisme. 
4 3) «La fabrication de la sociologie et d'autres disciplines». La consolidation de la sociologie comme discipline renvoie à trois penseurs : «l'outsider prolifique Gabriel Tarde, l'organisateur omniprésent René Worms, et l'universitaire rigoureux Émile Durkheim » (p. 67). Durkheim se brouille avec Tarde sur la question du réalisme social, et, en fondant L'Année sociologique, il se met directement en concurrence avec Worms, qui dirige la Revue internationale de sociologie. Des trois, Durkheim est celui qui reprend le programme de Comte afin de créer une sociologie scientifique (p. 76). Il est le premier à proposer des règles de méthode, à définir l'objet de la sociologie - les faits sociaux - et à utiliser la statistique, notamment la méthode des variations concomitantes dont son ouvrage sur le suicide en sera l'exemple le plus abouti. L'aventure de L'Année illustre aussi une nouvelle façon de travailler, fondée sur la coopération entre de jeunes intellectuels que Durkheim recrute pour recenser la littérature internationale: on les appelle désormais les durkheimiens. Ce travail de groupe n'implique pas une homogénéité intellectuelle, les collaborateurs de Durkheim restant libres d'exprimer leurs divergences, voire de quitter le groupe. Le champ de la sociologie naissante fait donc apparaitre une concurrence surtout entre Worms et les durkheimiens, concurrence qui contribue à définir la sociologie comme discipline scientifique et à la faire entrer à l'Université.

5 4) «Les métamorphoses des durkheimiens ». Heilbron rend compte de la manière dont les durkheimiens évoluent de la mort de Durkheim jusqu'à la Seconde Guerre mondiale. Il oppose à la thèse du déclin du durkheimisme la thèse opposée de son expansion : "Dans un nombre important de champs académiques, les idées durkheimiennes fleurissent et elles contribuent de manière significative à rénover la recherche " (p. 93). Malgré des divergences croissantes, tous les durkheimiens parviennent à transmettre l'œuvre de Durkheim aux générations suivantes, notamment en occupant des positions clefs dans le champ universitaire - Strasbourg, la Sorbonne, le Collège de France. Mais ces divergences auront eu raison de la cohésion du groupe qui se désagrège peu à peu selon plusieurs lignes de partage (pour ou contre la philosophie, recherche ou professorat, participation ou non aux Annales sociologiques qui succèdent à L'Année). Les nouveaux venus sont tentés de faire carrière hors de l'université (voir Georges Friedmann, Georges Duveau, Jean Stoetzel, les membres du Collège de sociologie). «Le durkheimisme devient un symbole de tout ce qui doit être rejeté, car démodé et conservateur » (p.114) et les étudiants s'en éloignent de plus en plus jusqu'en 1930. Si la sociologie durkheimienne survit dans l'entre-deux guerre, c'est en particulier grâce aux chercheurs, qui exercent néanmoins en dehors de la discipline stricto sensu.

6 5) «Pionniers par défaut?». Dans les années 1950, il est difficile de se faire sociologue en France. D'une part, les institutions académiques doivent être reconstruites. D'autre part, le champ intellectuel est investi par les philosophes, tel Jean-Paul Sartre pour qui "la sociologie de Durkheim est morte » (p.129). Enfin, la sociologie américaine impose une orientation très empirique. Georges Gurvitch tente de relancer la sociologie française contre cette tendance. Revenu de son exil aux États-Unis, il crée les Cahiers internationaux de sociologie puis fonde, en 1958, l'Association internationale des sociologues de langue française. Il contribue aussi à la fondation du Centre d'études sociologiques (1946), institution que Friedmann transformera en un laboratoire de recherches analogue à ceux du CNRS. Malgré ces efforts, la sociologie française se développe dans un sens de plus en plus empirique, tournée vers la question de la classe 
ouvrière et de ses conditions de travail (voir les travaux de Paul-Henry Chombart de Lauwe, p. 143 et suiv.). Ces sociologues d'après-guerre sont soutenus par les historiens des Annales (Fernand Braudel, Lucien Febvre, Marc Bloch), qui encouragent l'échange interdisciplinaire et croient au renouveau du durkheimisme.

7 6) «Cycles d'expansion et transformations du champ ». Les années 1960 marquent le début d'une expansion qui se fait en deux temps. Une première phase court de 1960 au milieu des années 1970. C'est le temps des grands récits, marqué par des intellectuels célèbres, tels Claude Lévi-Strauss, Louis Althusser, Michel Foucault, Roland Barthes, etc. Le structuralisme devient un espace fédérateur. Or ce mot recouvre une grande variété de programmes de recherche et il fera de ce fait l'objet de nombreuses critiques. Cette première phase stagne dès les années 1970 jusqu'au début des années 1980, en raison de facteurs contextuels. Les étudiants se détournent des idéaux des années 1960-1970 et optent pour des disciplines pouvant les mener à trouver un travail (p.159). Ceci pousse les intellectuels à s'engager pour la spécialisation de leur discipline sans toutefois rompre leur lien à la société civile (voir l'exemple emblématique de Bourdieu dans les années 1980-1990). Cette transformation du champ intellectuel marque une seconde phase d'expansion de la sociologie. Au plan institutionnel, elle se caractérise par une augmentation des postes académiques - Heilbron cite ici notamment les cas du CNRS et de l'EHESS (p. 164 et suiv.). Au début des années 2000, le métier de sociologue en France a bien changé. Les chercheurs doivent à la fois desservir une population étudiante qui augmente, tout en recherchant eux-mêmes des financements pour leur activité de chercheur. De ces deux profils, celui du chercheur transdisciplinaire dominerait la discipline aujourd'hui.

7) «Styles intellectuels et dynamique des groupes de recherche ». Heilbron conclut son ouvrage sur la présentation des groupes et des styles de travail de certaines grandes figures de la sociologie française contemporaine, à savoir Alain Touraine, Michel Crozier, Raymond Boudon et Pierre Bourdieu. Touraine et Crozier ont porté leur attention sur l'industrie, le travail et les classes ouvrières. Touraine va évoluer vers une sociologie des nouveaux mouvements sociaux et travaille à une approche historienne des transformations de la société contemporaine. Crozier tire profit de sa connaissance de la sociologie américaine pour développer une sociologie des organisations. Il vise à réformer la bureaucratie, avant d'évoluer vers une sociologie du management et des organisations privées. Boudon est le sociologue de l'individualisme méthodologique, qu'il tente de populariser en France. En même temps, il rétablit un programme tourné vers les pères fondateurs français et allemands de la discipline. Bourdieu se distingue non seulement par les nouvelles institutions qu'il crée - comme les Actes de la recherche en sciences sociales -, mais aussi par le fait de traverser les disciplines et d'y appliquer son système théorique.

9 Le livre de Heilbron est un voyage très documenté dans plus de 150 ans de sociologie française. Avoir réussi à exprimer en un nombre modeste de pages les lignes directrices de cette tradition relève de la performance. Le choix de ne pas s'en tenir aux seuls auteurs et à leurs théories, mais de considérer les institutions dominantes, les organes de publication, les fluctuations du nombre d'étudiants, le contexte global, offre un regard original. Par son caractère pédagogique, ce livre sera d'une aide précieuse pour tout étudiant désirant mieux connaître la sociologie française. Certes, il ne faut pas en attendre un panorama exhaustif - ce n'est pas son ambition. Il s'agit avant tout de proposer des orientations pour mieux comprendre comment la discipline s'est 
structurée en France. L'application de la théorie des champs de Bourdieu remplit bien cette fonction, même si elle a parfois ses inconvénients. Les différents courants sociologiques sont souvent exposés de façon très polarisée - Comte vs le champ des sciences humaines françaises au XIx ${ }^{\mathrm{e}}$ siècle, Durkheim vs Worms, Bourdieu vs Touraine/ Crozier/Boudon. Cela donne l'impression que la sociologie française fonctionne comme un monopole où les sociologues se battent entre eux pour imposer leur orientation. Ces luttes existent à n'en pas douter, mais elles sont loin d'épuiser l'histoire que Heilbron relate. Car, nous dit-il, la sociologie française est avant tout un champ diversifié qui se cherche à la croisée d'autres disciplines, situation qui dans les moments de crise lui profite. Elle tire ici ses ressources de ses voisins, là leur sympathie et, dans ces échanges intra- et interdisciplinaires, non seulement elle survit aux siècles, mais elle se transforme en tant que pratique scientifique.

L'épilogue du livre nous permet enfin d'évoquer des questions abordées dans l'ouvrage trop rapidement. Ainsi, à propos des influences d'autres traditions sur la sociologie française, Heilbron parle-t-il surtout des États-Unis. S'il est certain que son livre vise d'abord un public anglophone, on serait tout de même tenté de se demander ce qu'il en est des autres traditions ayant influencé - et réceptionné - la sociologie française de manière décisive, comme l'Allemagne par exemple. Quant à la thèse de la double expansion de la sociologie française de 1960 à nos jours, elle peut encore être discutée. Si l'analyse de la première phase (1960-1970/1980) convainc, celle de la seconde phase n'emportera pas forcément l'assentiment général. Heilbron y parle en effet avant tout de quelques institutions parisiennes prestigieuses à laquelle la sociologie en France ne se réduit pas. De surcroît, il ne considère pas l'impact des différentes réformes mises en place dès la fin des années 1990. Mais c'est aussi parce que ces points restent ouverts au débat que ce livre encourage le lecteur à s'intéresser à la manière dont la sociologie française s'est structurée dans la longue durée - et c'est là également l'un de ses mérites.

\section{AUTEURS}

\section{CHRISTIAN PAPILLOUD}

Martin-Luther-Universität Halle-Wittenberg - Institut für Soziologie 